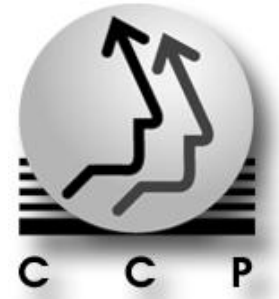

Población y Salud en Mesoamérica

Revista electrónica publicada por el

Centro Centroamericano de Población,

Universidad de Costa Rica, 2060 San José, Costa Rica

http://ccp.ucr.ac.cr

Población y Salud en Mesoamérica

Revista electrónica semestral, ISSN-1659-0201

Volumen 11, número 1, artículo 2

Julio - diciembre, 2013

Publicado 1 de julio, 2013

http://ccp.ucr.ac.cr/revista/

\title{
Esperanza de vida entre 1991-2011: explicación epidemiológica a la desigualdad por género en Tarragona, una provincia de Cataluña, España
}

\author{
Margarita Gonzalvo-Cirac \\ Montserrat Girabent Farrés \\ María Victoria Roqué Sánchez
}

\section{(cc) $\mathrm{BY}-\mathrm{NC}-\mathrm{ND}$}

Protegido bajo licencia Creative Commons

Centro Centroamericano de Población 


\title{
Esperanza de vida entre 1991-2011: explicación epidemiológica a la desigualdad por género en Tarragona, una provincia de Cataluña, España
}

\section{Life Expectancy between 1991-2011: Epidemiological Reason for the Gender Inequalities in the Province of Tarragona (Catalonia-Spain)}

\author{
Margarita Gonzalvo-Cirac ${ }^{1}$, Montserrat Girabent Farrés ${ }^{2}$, María Victoria Roqué Sánchez ${ }^{3}$
}

\section{RESUMEN}

El objetivo de esta investigación es determinar si las distintas causas de muerte explicarían la desigualdad de la mortalidad por sexo entre 1991 y 2011 en la provincia de Tarragona (Cataluña, España) en edades avanzadas. Con las defunciones por edad y por sexo del Instituto Nacional de Estadística español (INE) y de Cataluña (IDESCAT) se realizan tablas de mortalidad para describir la evolución de la mortalidad. Con las causas de muerte se elabora una agrupación homogénea de grandes causas. La metodología utilizada será la estandarización indirecta que facilita la interpretación exacta de la clasificación de las enfermedades mortales y la comparación entre territorios. Los resultados muestran que la esperanza de vida entre sexos difiere 6 años, más alta en las mujeres que en los hombres, sin embargo, por primera vez en el siglo XX, el aumento en años es mayor en los hombres. En cuanto a las causas de muerte, Tarragona tiene menor mortalidad que España en $1991(0,71$ y 0,75) y para las mujeres en 2001 $(0,90)$ y una mayor incidencia de muertes provocadas por el conjunto de "otras enfermedades" (mayor que 1): enfermedades degenerativas, mentales, nerviosas, endocrinas, etcétera. Parece que las políticas de salud pública tendrían que priorizar más los avances en longevidad, especialmente en las mujeres, pues ellas son las que alcanzan mayor esperanza de vida al nacer y crean nuevos patrones epidemiológicos -desarrollan nuevas enfermedades- actualmente mentales y nerviosas.

Palabras clave: España, Tarragona, esperanza de vida, epidemiología, mortalidad, identidad de género, salud mental.

\footnotetext{
${ }^{1}$ Universitat Internacional de Catalunya. ESPAÑA. mgonzalvocirac@gmail.com

${ }^{2}$ Universitat Internacional de Catalunya. ESPAÑA. mgirabent@uic.es

${ }^{3}$ Universitat Internacional de Catalunya. ESPAÑA. mvicro@gmail.com
} 


\begin{abstract}
The objective of this research is to determine if different causes of death would explain gender inequality in mortality at advanced ages between 1991 and 2011 in the province of Tarragona (Catalonia, Spain). Mortality tables are made based on deaths according to age and sex from statistics provided by Instituto Nacional de Estadística español (INE) and Institut d'Estadística de Catalunya (IDESCAT) in order to show the evolution of mortality. From this, a homogenous group of the main causes of death is produced. Indirect standardization is the methodology used to carry out an exact interpretation of the classification of deadly diseases and comparison between areas. The results show that life expectancy differs between sexes by six years, with women having a greater life expectancy. However, for the first time in the twentieth century, male life expectancy grows more than female life expectancy. As for causes of death, Tarragona has lower mortality than Spain in 1991 (0.71 and 0.75) and for women in 2001 (0.90) and a higher incidence of deaths from the set of "other diseases" (greater than 1): new degenerative diseases, mental, nervous and endocrine illnesses, etc. Public health policies should give more priority to advances in longevity, especially in women. They reach the highest levels of life expectancy from birth and create new epidemiological patterns. Additionally, they develop new diseases, such as mental and nervous illnesses at the moment.
\end{abstract}

Key words: Spain, Tarragona, life expectancy, epidemiology, mortality, gender identity, mental health.

Recibido: 23 feb. 2013

Aprobado: 28 mayo 2013 


\section{INTRODUCCIÓN}

La evolución de la mortalidad en España entre 1991 y 2011 se enmarca en una situación demográfica peculiar: el gran crecimiento de la población por longevidad. La esperanza de vida no para de crecer (Blanes, 2007; Cabré y Domingo, 2007; Vallin y Meslé, 2010; Jagger, 2013). En 1991 la vida media para los hombres era de 73,40 años y para las mujeres de 80,44 años; alcanzó en 2011 78,70 años para el sexo masculino y 84,70 para el femenino. Muy parecido recorrido presenta la provincia catalana de Tarragona: la esperanza de vida al nacer en 1991 para los hombres es de 73,88 y para las mujeres de 80,33; mientras que en 2011, ellos alcanzan 78,45 años y las mujeres, 84,70.

Esta desigual mortalidad por sexo no acaba de justificarse actualmente cuando los estilos de vida, el acceso a los servicios sanitarios, etcétera, entre la población no difieren según el género y se igualan los comportamientos (Regidor, 2002). Según este mismo autor estas diferencias tendrían que tener una tendencia al descenso. Fernández y Méndez (2007) señalan para Costa Rica unos más igualados estilos de vida para los hombres y para las mujeres pero en edades muy avanzadas.

Otros autores (Mesle y Vallin, 2002; Blanes, 2007; Blanes y Spijker, 2010; Vallin y Meslé, 2010; Gonzalvo-Cirac y Albareda, 2013) señalan que la clave para entender esta desigual esperanza de vida por sexo se encuentra en la diferente causa de muerte en las edades avanzadas para los hombres y para las mujeres más que en un comportamiento similar por sexo. En este contexto se sitúa esta investigación local. El objetivo es determinar qué causas de muerte fueron responsables en la provincia de Tarragona de la mortalidad por sexo y qué explicaría la diferente evolución de la esperanza de vida al nacer entre sexos. Para ello se comparan las causas de muerte de Tarragona con otra que tenga una tendencia similar en cuanto al comportamiento de la evolución de la esperanza de vida, en nuestro caso España.

A través del marco conceptual de la Teoría de la Transición Epidemiológica se expondrá cuál es la evolución epidemiológica y lanzaremos resultados novedosos sobre si la provincia de Tarragona ha pasado de la Tercera o incluso la Cuarta Fase de la Transición Epidemiológica, a las fases sucesivas, como han descrito autores como Horiuchi (1999) y Meslé y Vallin (2002) para explicar la evolución de las causas de muerte en la zona de estudio.

La evolución de la mortalidad a edades avanzadas iría ligada al aumento de enfermedades infecciosas -pese a la disminución de la incidencia del SIDA (Blanes, 2007)-; al descenso de las enfermedades mortales cardiovasculares (Mora García, 2012), aunque con una ralentización de su caída en los últimos años; al aumento de cánceres en edades maduras, aunque con reducción de los niveles de ciertos tipos de cáncer (pulmón, mama) (Machón, 2012); y al aumento de enfermedades degenerativas propias de esas edades -sobre todo entre las mujeresespecialmente de las catalogadas como "otras causas no transmisibles" (Vallin y Meslé, 2010): las producidas por trastornos mentales (Alzheimer y otras formas de demencia), las del sistema nervioso y las de los huesos, las producidas por los órganos genitourinarios, las endocrinas, las de la piel, las de la sangre, etcétera.

Las conclusiones señalan cuáles han sido las razones del comportamiento de la distinta esperanza de vida entre hombres y mujeres en la provincia de Tarragona. 


\section{MÉTODOS Y DATOS}

El inicio de la construcción de la teoría de la Transición Epidemiológica fue propuesto por R. Omram en 1971. El concepto se acuña para describir el descenso de la mortalidad a través de las diferentes enfermedades que afectan en cada tiempo a la población y, desde el principio Omram (1971) sugiere tres primeras fases. Estas fases tendrían una cronología en países desarrollados hasta aproximadamente 1970-80. La modificación de unas causas de muerte a otras ha recibido el nombre de cambio de patrón epidemiológico.

Poco a poco, se sugiere la formulación de una Cuarta Fase (Olshansk y Ault, 1986, y Olshansky, 1998), denominada así por la aparición de enfermedades degenerativas a edades tardías y caracterizada por:

1. el retraso progresivo de las edades en que las principales enfermedades degenerativas circulatorias, respiratorias, cáncer, entre otras- provocan la muerte,

2. la aceleración de los avances médicos y la reducción de los factores de riesgo en un contexto caracterizado por el envejecimiento de la población,

3. y, gracias a la implantación de vastos programas de salud, la mejora de servicios sanitarios (Bah y Rajulton, 1992; Pérez-Brignoli, 2010).

Al mismo tiempo, Rogers y Hackenberg (1987) sugieren otra Cuarta Fase a la que denominan fase híbrida y cuya característica principal sería un cambio en los patrones de mortalidad debido a la mayor influencia de los hábitos humanos sobre los factores de riesgo. Esta se caracterizaría por:

1. un descenso de la mortalidad a edades muy tardías. Las enfermedades infecciosas todavía no se han erradicado y se encuentran elevadas puntas en grupos de población dentro de las mismas zonas geográficas donde la mortalidad es muy baja;

2. los llamados vicios o malos hábitos, como el tabaquismo, sedentarismo, alcoholismo, etcétera, aumentan el riesgo de sufrir enfermedades como cánceres y problemas cardiovasculares generales, y

3. y los hábitos personales y los comportamientos individuales adquieren importancia sobre la disminución de la morbilidad y de mortalidad.

La primera cuarta etapa, según Bah y Rajulton (1992), se centra en los factores explicativos macro relacionados con los servicios sanitarios, los programas de salud, etcétera, mientras que la segunda cuarta etapa se centra más en los comportamientos personales e individuales. Puede establecerse que ambas etapas descritas sean complementarias y puedan combinarse bien para describir, en general, la Cuarta Fase de la Transición Epidemiológica, en la que habría entrado España hacia 1980.

Una Quinta Fase de la Transición Epidemiológica aparecería, caracterizada por el descenso de enfermedades degenerativas hasta entonces principales: las cardiovasculares (ya descritas por Meslé y Vallin) y los tumores (por Horiuchi, 1999), las cuales se superponen.

La Sexta Fase se describe por una mortalidad y morbilidad concentrada en edades muy avanzadas y dominada por enfermedades del aparato respiratorio (neumonías, bronquitis, entre 
otras), por enfermedades mentales y del sistema nervioso, y por otras de difícil catalogación por la multiplicidad de factores que intervienen en la muerte. Es la etapa denominada "slowing of senescence", que supone un punto y seguido en el esquema epidemiológico transicional creado hace 40 años por Omram.

Esta sucesión de fases, cuartas, quinta y sexta fases, son descritas por Horiuchi y por los investigadores Vallin y Meslé (2010), como alternancia en la preponderancia de unas enfermedades a otras, lo que alarga muy poco la esperanza de vida, ya que los años de vida ganados se concentran en edades cada vez más avanzadas.

Unas de las fuentes utilizadas en esta investigación hacen referencia a los datos de Población (en los Censos) y otras, a los Fallecimientos y sus Causas (publicados en el Movimiento Natural de la Población). La descripción de la mortalidad se realiza a través de la esperanza de vida al nacer $\left(\mathrm{e}_{0}\right)$ y a la edad 65 años, indicador preciso para resumir el análisis de la mortalidad actual. La información de estas fuentes de variables ha sido extraída de los datos publicados por el Instituto Nacional de Estadística (INE) y del Institut d'Estadística de Catalunya (IDESCAT).

Para el análisis descriptivo por causas de muerte se ha construido una serie homogénea de grandes causas de muerte, que parte de la información existente en la novena y décima revisión de la Clasificación Internacional de Enfermedades (CIE). Apenas ha repercutido el cambio de clasificación, ya que la agrupación ha sido para 10 grandes causas de muerte. Además se han revisado los trabajos de agrupación de causas de muerte por otros autores (Casselli, Mesle y Vallin, 2002; Blanes, 2007; Vallin y Mesle; 2010) y finalmente se ha validado la agrupación diseñada de grandes causas de muerte combinación entre el modelo etiológico y el anatómico: un gran conjunto de enfermedades infecciosas, otro de cánceres y tumores malignos que prioriza estas dos primeras agrupaciones a las siguientes tres, según la enfermedad en los aparatos circulatorio, respiratorio y digestivo. Continúan las enfermedades puerperales, la debilidad congénita y las afecciones perinatales. Finalmente, tres grandes cajones con las enfermedades mal definidas (difícil clasificación en la CIE), las muertes violentas (aumento el suicidio) y el conjunto "otras enfermedades": mentales, nerviosas, de la sangre, de los huesos, endocrinas, piel y pequeñas enfermedades mortales no clasificadas anteriormente y que en este período han ido alcanzando protagonismo.

El método de análisis utilizado es la estandarización indirecta, reconocido como el mejor método para el estudio descriptivo de la mortalidad a nivel epidemiológico (Nordness, 2006). La explicación más plausible para el uso de la estandarización (directa o indirecta) es que la tasa bruta de mortalidad se encuentra muy influenciada por la estructura por edades de la población, lo cual plantea problemas y equívocos a la hora de comparar e interpretar los resultados, los aumenta o reduce, de tal forma que tiene que utilizarse un indicador menos sesgado, como es el caso de la estandarización. Una característica importante es que se elimina el efecto que pudiera tener la estructura por edades a la hora de comparar las tasas entre distintas zonas geográficas y diferentes períodos.

En la investigación que se presenta se utiliza la estandarización indirecta. Este método de ajuste está basado en promedios ponderados y así se facilita la comparación de dos poblaciones con diferente estructura por edad. Como explican Nordness (2006) y Schoenbach (2000), es uno de los métodos más utilizados para el estudio de las causas de muerte en epidemiología y en salud 
pública por ser sencillo de cálculo, básico y estable. En el caso de la estandarización indirecta el patrón de comparación utilizado es denominado ley o pauta, las tasas específicas de mortalidad. Con lo que la investigación epidemiológica actual suele presentar sus resultados a través de este método que consiste en aplicar una misma serie de tasas específicas de mortalidad a dos o más poblaciones con diferente estructura de edad, caso que no ocurre con la estandarización directa.

El análisis de estandarización indirecta muestra un único resultado que recibe el nombre de Índice de Mortalidad Estandarizado (IME) o "Standardized Mortality Ratio (SMR)", en nuestra investigación, muestran el nivel de la provincia analizada en relación con el patrón elegido, en este caso España.

La estandarización indirecta estima cuán diferente sería la mortalidad de una población "X" si, en lugar de morir de acuerdo a su patrón de mortalidad por edad característico, sus efectivos de población (los que constan en la pirámide) murieran de acuerdo a otra ley de mortalidad conocida (la tasa estándar o tipo específica de mortalidad por edad). La metodología consiste en aplicar sobre la pirámide de población de la zona geográfica que estamos analizando una ley de mortalidad estándar y comparar las defunciones que se generan (esperadas) con las que realmente se han producido (reales).

La mortalidad tipo de este trabajo de investigación es la del conjunto de España: una serie de tasas específicas de mortalidad de España serán la ley o pauta, la mortalidad estándar. Estas tasas (=pauta) se aplican a la estructura de la población de la provincia de Tarragona, con lo que se obtiene un número de defunciones esperadas, las cuales, sumadas, serán el denominador para el cálculo de los IME

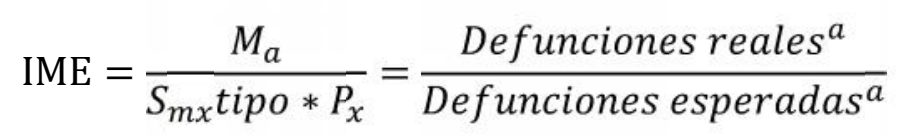

- si el IME > 1 la mortalidad observada en Tarragona por dicha causa es mayor que la esperada si la provincia tuviera la pauta de mortalidad por edad española para dicha causa;

- $\quad$ si el IME < 1 significa, por el contrario, que la mortalidad de Tarragona es menor para esta causa que la de España.

La interpretación de los resultados, los IME, es un poco restringida, ya que solo se pueden comparar los IME del área investigada con respecto a los IME del área de la cual se ha tomado la tasa tipo, en este caso, Tarragona con respecto a España. Sin embargo, esta característica es muy importante para los estudios geográficos y territoriales, ya que es uno de los métodos más fiables, al ampliar la información de la zona elegida, el análisis se puede hacer con más profundidad y refuerza mejor la importancia de los factores espaciales o geográficos en el conjunto de lo que se quiere investigar. 
Los indicadores de mortalidad de este estudio se han elaborado sobre los fallecidos para períodos de cuatro años centrados sobre los años censales, en 1991 y en 2001. En el caso del año 2011, como no ha habido censo por el impacto de la crisis económica (INE), se han construido unos datos seguros y robustos de población con el Padrón de 2005 y los datos publicados por el INE y por el IDESCAT para el 2012. Obviamente, para los años censales 1991 y 2001, se han tomado las defunciones de los dos años anteriores y posteriores al momento de referencia del censo y, para el recuento de población de 2011, se han tenido en cuenta las defunciones de 2008, 2009, 2010 y 2011.

\section{RESULTADOS}

Una rápida descripción de la evolución de la mortalidad muestra que, desde el inicio del siglo XX, es a partir de 1991 cuando aumenta más la esperanza de vida masculina que la femenina. La diferencia marca un novedoso cambio de tendencia (tabla 1). Ganancia debida en gran parte a la reducción de la sobremortalidad en los hombres jóvenes, muy importante todavía en 1991 (Blanes, 2007).

En cuanto a la diferencia de esperanza de vida entre hombres y mujeres, continúa siendo amplia, es de 6 años. La provincia es la que conserva mayor diferencia en 2011. En este sentido, la esperanza de vida al nacer masculina en 2011 (un poco menos de 79 años) se iguala a la que poseían las mujeres en 1981 (cerca de 79 años).

La principal característica de esta evolución es la progresiva concentración de las ganancias de años en edades cada vez más avanzadas (tabla 2: elaboración propia a partir de los datos publicados para España y Cataluña [Cabré y Domingo, 2007], para España y provincias [Blanes, 2007] y cálculos propios). Aumento mayor en las mujeres que en los hombres. La mayor supervivencia es un factor clave del envejecimiento de la población, al tiempo que el crecimiento de la población se ha beneficiado de esta mejora en la longevidad.

Un estudio básico sobre el porcentaje de defunciones por grandes causas de muerte en la provincia de Tarragona (tabla 3) señala el descenso de las enfermedades degenerativas "tradicionales" (aparato circulatorio y cáncer) en los primeros años del siglo XXI, con más intensidad en los hombres que en las mujeres. Las causas respiratorias aumentaron su incidencia desde 1970, pero se han frenado desde el año 2000; por el contrario, el conjunto de "otras enfermedades" que incluyen enfermedades degenerativas de edades avanzadas (mentales, del sistema nervioso, de huesos) están aumentando rápidamente en la última década (Ramos, 2012), especialmente entre las mujeres, propias del "retraso de la senectud" (Olshansky, S. J. y Ault, 1986).

Observamos ahora si esta evolución de la mortalidad demográfica, a edades cada vez más avanzadas y con una diferencia entre sexos de 6 años, en Tarragona, tiene su reflejo en las diferencias de patrones epidemiológicos y si difieren del patrón elegido España, que tiene un comportamiento similar en la evolución de la esperanza de vida.

Al comparar los Índices de Mortalidad Estandarizados (IME) de Tarragona y España se observa en las tres estandarizaciones indirectas realizadas (tabla 4): la mortalidad de Tarragona es 
inferior a España en 1991 para ambos sexos y para las mujeres en 2001; mientras que es igual para los hombres en 2001 y para ambos sexos en 2011.

a) Estandarización del censo de 1991: los IME presentan una menor mortalidad para prácticamente la totalidad de las causas de muerte, especialmente en circulatorias, respiratorias, y cáncer-tumores. Sólo en la agrupación "otras enfermedades" y en las causas externas que afectan a las mujeres la situación de la provincia es peor; dentro de la agrupación "otras enfermedades" se recogen las provocadas por enfermedades de la piel, nutricionales, mentales, nerviosas, entre otras. Cabe señalar que este conjunto de causas son cada vez más altas y distancian las diferencias entre hombres y mujeres, y entre áreas geográficas. Baja incidencia tienen las enfermedades mortales de las afecciones perinatales en los dos sexos, las del aparato respiratorio (favorable para las mujeres) y las del aparato digestivo (favorable para los hombres).

b) Estandarización del censo de 2001: los IME ofrecen unos resultados muy semejantes a los obtenidos en 1991. La incidencia de la mortalidad en Tarragona es menor para la gran mayoría de las causas de muerte, aunque difiere en que los niveles están ahora más cercanos a los españoles. Otra diferencia que encontramos es que en la provincia hay mayor mortalidad en los dos sexos (y no solo en las mujeres) por causas externas, una tendencia que ya se daba en Tarragona en décadas anteriores, debido a su inclusión en el eje de provincias con mayor nivel de motorización (García y Gadea, 2004, y Blanes, 2007). Finalmente, también es mayor la mortalidad en la provincia que en España en el conjunto de "otras enfermedades" para ambos sexos.

c) Estandarización en el año 2011: los resultados, que para el conjunto de las defunciones muestran mortalidades muy parecidas para ambos sexos y repiten a grandes rasgos los patrones epidemiológicos de 1991 y 2001, aunque con valores más similares a los estándares, son más cercanos a 1. De nuevo la mortalidad causada por el conjunto de "otras enfermedades", las de causas externas, las "mal definidas" en ambos sexos y las respiratorias en mujeres, tienen mayor incidencia en Tarragona que en España. El resto de causas siguen ocasionando menos muertes en la provincia.

El hecho más significativo es la gran incidencia en la provincia de las muertes provocadas por el conjunto de "otras enfermedades" durante todo el período de estudio. La evolución se relaciona con el crecimiento de nuevas enfermedades degenerativas, mentales, del sistema nervioso, etcétera, que afectan particularmente a las edades más avanzadas y que generan un nuevo cambio de patrón epidemiológico (¿sexta fase?), al tiempo que es un tema con profundas implicaciones de política sanitaria y asistencial, de sumo interés para las administraciones públicas, ONGs y organismos privados.

\section{DISCUSIÓN}

Los avances en la longevidad con consecuencias en el crecimiento de la población y en la estructura por edades (mayor envejecimiento) constituye uno de los aspectos esenciales de la evolución de la mortalidad en el período 1991-2011 (Blanes, 2007; Cabré y Domingo, 2007; Ramos, 2012; Prioux y Barbieri, 2012). 
Lo novedoso de la investigación es que este aumento de esperanza de vida diferente entre hombres y mujeres se justifica por las distintas enfermedades a las que mueren las personas del sexo masculino y las del femenino. Según Regidor y su equipo de investigadores (2002), tendría que haber una tendencia al descenso entre las diferencias por sexo, sin embargo los resultados de esta investigación demuestran lo contrario. Si bien es cierto que entre 1991-2011 es mayor el aumento de la esperanza de vida masculina con respecto a las mujeres, la diferencia de la vida media entre nacer hombre o mujer y sobre todo entre cumplir 65 años siendo hombre o mujer se dilata.

En este sentido Regidor (2002) argumenta que esta tendencia a unificarse la esperanza de vida entre sexos es debido a los cambios en los estilos de vida que igualan los comportamientos entre hombres y mujeres, etcétera. Tal explicación, podría ser cierta a edades jóvenes, sobre todo por la disminución de la mortalidad por causas externas, que afecta directamente a la población de edades jóvenes-adultas, como también señala Blanes (2007) en su estudio. En nuestra investigación encontramos, sin embargo, enfermedades mortales diferentes para los hombres y para las mujeres a edades avanzadas. Quizá, aunque no forma parte del estudio, podríamos encontrar comportamientos similares, no tanto en edades jóvenes, sino en edades ancianas; como sugieren Fernández y Méndez (2007) para Costa Rica, podría ser que la esperanza de vida, las causas de muerte y los estilos de vida a partir de los 80 años de edad entre hombres y mujeres sean más parecidos.

Son escasas las investigaciones sobre la relación esperanza de vida y enfermedades mortales a edades avanzadas en Cataluña, siendo esta investigación una contribución necesaria.

Los resultados sobre las enfermedades mortales confirman las características principales de los cambios de salud y la evolución de la morbilidad que caracterizan a las fases cuarta, quinta y sexta de la Transición Epidemiológica (explicada en el apartado 2): aumento de la mortalidad por enfermedades infecciosas, descenso de las enfermedades mortales cardiovasculares (Mora García, 2012), aumento de cánceres (Machón, 2012) y aumento de la mortalidad por el conjunto de "otras enfermedades", especialmente en edades muy avanzadas y sobre todo en las mujeres. Este conjunto de causas mortales es denominado "otras causas no transmisibles" (Vallin y Meslé, 2010) y la mayoría son producidas por trastornos mentales (Alzheimer y otras formas de demencia), las del sistema nervioso y las de los huesos, las producidas por los órganos genitourinarios, las endocrinas, las de la piel, las de la sangre, etcétera.

Esta evolución de los patrones epidemiológicos en la provincia de Tarragona con respecto a España parece indicar que existe una sucesión de fases transicionales (como señalan Vallin y Meslé [2010] en sus estudios) distinta por sexo y con calendarios cada vez más cortos -y más adelantado en la provincia que el español-. La mortalidad de los hombres de Tarragona parece haber entrado en la Quinta Fase transicional a inicios del siglo XXI, en la que el descenso del cáncer y de las enfermedades cardiovasculares sería el principal marcador respecto a la etapa precedente, mientras que la mortalidad de las mujeres de Tarragona inicia la Sexta Fase, con mayor incidencia de las causas de muerte cerebrovasculares (dentro de las circulatorias), las mentales y las del sistema nervioso, en otras palabra, de las propias del "retraso de la senectud" según Horiuchi (1999). De ahí el indicador más alto en los IME. 


\section{CONCLUSIONES}

Diferentes enfermedades son causas de muerte entre hombres y mujeres a edades muy avanzadas, lo cual determina que la distancia entre la esperanza de vida entre sexos puede deberse principalmente a este hecho. La investigación realizada sobre la provincia de Tarragona entre 1991-2011 subraya esta realidad.

Como reflexión final, la estandarización indirecta parece que es un buen indicador para medir las transformaciones demográficas de la población (la esperanza de vida) y su impacto en la salud a pesar de que solo se pueden comparar dos áreas geográficas analizadas, los IME del área investigada con respecto a los IME del área de la cual se ha tomado la tasa tipo, en este caso, Tarragona con respecto a España. Destaca, sin embargo, el uso de la estandarización indirecta para dar realce a los estudios geográficos, territoriales y epidemiológicos al ampliar la información de la zona elegida y hacer más exacta la interpretación al mejorar el análisis y los resultados. En esta investigación, las tendencias recientes encontradas de la evolución de la mortalidad (transición demográfica) se adecuan a los rasgos que definen y caracterizan las últimas fases de la teoría de la transición epidemiológica.

El papel de la administración pública y sus políticas sanitarias están incidiendo en los factores de riesgo de tipo individual y colectivo, para conseguir una mejor calidad de vida con la que llegar a edades avanzadas. Este también ha sido el camino para conseguir los resultados en esperanza de vida en Costa Rica y otros países (Pérez-Brignoli, 2010, y Pélaez-Herreros, 2012). Estas políticas se tendrán que centrar en aquellas causas de muerte que están ganando peso y que tendrán más importancia en el futuro, como las enfermedades degenerativas mentales y del sistema nervioso que afectan a la población de edad más avanzada, con especial atención en las mujeres ancianas que actúan como pioneras en la experimentación de los nuevos patrones demográficos (mayor esperanza de vida) y epidemiológicos (nuevas enfermedades). También podría generar, debido a la crisis actual, un aumento de enfermedades infecciosas, respiratorias, en estas poblaciones más vulnerables.

En este sentido, la mayoría de los estudios sobre la mortalidad actual, tanto desde el punto de vista demográfico como epidemiológico, ha mostrado resultados para edades jóvenes adultas y en especial las masculinas, pero faltan estudios sobre edades avanzadas y sobre el papel femenino. Las mujeres alcanzan los niveles máximos de esperanza de vida al nacer e innovan nuevas enfermedades mortales (actualmente mentales y nerviosas) a edades muy avanzadas. Creando ellas los actuales patrones epidemiológicos y las sucesivas fases de la transición epidemiológica.

El estudio de la mortalidad constituye un elemento clave para ampliar los conocimientos sobre salud, especialmente sobre salud pública. Las políticas dirigidas a la reducción de las desigualdades sociales en salud y a una mayor calidad de vida en edades avanzadas todavía tienen mucho por estudiar y por revisarse, ya que impactan fuertemente en la sociedad, en la salud y en la misma población. 


\section{BIBLIOGRAFÍA}

Bah, S. y Rajulton, F. (1992). Has Canadian mortality entered the Fourth Stage of the epidemiologic Transition? Canadian Studies in Population (18), 18-41.

Blanes, A. (2007). La mortalidad en la España del siglo XX. Análisis demográfico y territorial. Tesis doctoral, Universitat Autònoma Barcelona, Bellaterra.

Blanes, A. y Spijker, J. (2010). Supervivència i patrons de mortalitat de la població catalana, 1960-2007. Treballs de la Societat Catalana de Geografia, 69, 67-95.

Cabré, A. y Domingo, A. (2007). Demografia i immigració, 1991-2005. Economia catalana: reptes de futur. BBVA, Departament d'Economia de la Generalitat Catalunya; Barcelona, 105-126.

Casselli, G., Mesle, F. y Vallin, J. (2002) Epidemiologic transition theory exceptions. En Workshop First Seminar of the IUSSP Committee on Emerging Health Threats: Determinats of Diverging Trends in Mortality, IUSSP/Max Planck Institute for Demographic Research.

García, A. y Gadea, R. (2004). Estimación de la mortalidad y la morbilidad por enfermedades laborales en España. Archivos de Prevención de Riesgos Laborales (7), 3-8.

Gonzalvo-Cirac, M. (2011). Las mujeres vivimos más. Concepto de salud y mortalidad diferenciada. Alemania: Editorial Académica Española.

Gonzalvo-Cirac, M. y Albareda, S. (2013). Epidemiología, medio ambiente y desigualdades por género en Tarragona, una provincia de Cataluña (España), 1960-1990. Población y Salud en Mesoamérica, 10, 2 (6), 1-17.

Fernández, X. y Méndez, E. (2007). Estilos de vida y factores de riesgo para la salud de las personas adultas mayores del proyecto CRELES-Costa Rica 2004-2006. Población y Salud en Mesoamérica, 5, 1-18.

Horiuchi, S. (1999). Epidemiological transitions in human history. En Chamie, J. y Cliquet, R. L. (eds), Health and Mortality. Issues of global concern. Proceedings of the symposium on health and mortality, Bruselas, 19-22 Noviembre.

Jagger, C., McKee, M., Christensen, K., Lagiewka, K., Nusselder, W., Van Oyen, H., Cambois, E., Jeune, B. y Robine, J. M. (2013). Mind the gap-reaching the European target of a 2-year increase in healthy life years in the next decade. Eur J Public Health first published online, 1-5.

Machón Sobrado, M., Mozo Carollo, I., Tobalina Gómez, M. C., Castro Laiz, V., Martínez Cobo, R., Izarzugaza Lizarraga, I., Larrañaga Larrañaga, N. (2012). Supervivencia del cáncer en el país vasco entre 1995 y 2004. Rev. Esp. Salud Pública, 86, 293-299.

Mesle, F. y Vallin, J. (2002). La transition sanitaire: tendences el prespectives. En Casselli G, Vallin J y Wunsch G. Demographie: analyse et synthèse. Les determinants de la mortalité, París, INED, 439-461.

Mora García, G., Salguedo Madrid, G., Ruíz Diaz, M., Ramos Clason, E., Alario Bello, A., Fortich, A., Mazenett, E., Gómez Camargo, D., Gómez Alegría, C. (2012). Concordancia entre cinco definiciones de síndrome metabólico. Cartagena, Colombia. Rev. Esp. Salud Pública, 86, 301-311.

Nordness, R. (2006). Epidemiología y bioestadística, Madrid: Medical.

Olshansky, S. J. y Ault, A. B. (1986). The fourth stage of the epidemiologic transition: the age of delayed degenerative diseases. En The Milbank Quarterly, 64, 355-391. 
Olshansky, S. J., Carnes, B., Rogers, R. y Smith, L. (1998). Emerging infectious diseases: the Fifth stage of the epidemiologic transition? En World Health Statistic Quarterly, 51, 207217.

Omram, R. (1971). The Epidemiologic Transition. A Theory of the Epidemiology of Population Change. En The Milbank Quarterly, 49, 509-538.

Pélaez-Herreros, O. (2012). Evolución de la esperanza de vida de Costa Rica en el contexto global (1930-2010). Población y Salud en Mesoamérica, 10 (3), 1-22.

Pérez-Brignoli, H. (2010). La población de Costa Rica, 1750-2000, una historia experimental, Costa Rica: UCR.

Prioux, F. y Barbieri, M. (2012). L'évolution démographique récente en France: une mortalité relativement faible aux grands ages. Population, 67, 597-656.

Ramos, M, A. (2012). Análisis de las principales variables determinantes de la evolución de la mortalidad por demencias en Andalucía. Rev. Esp. Salud Pública, 86, 219-228.

Regidor, E., Borrell, C., Pasarín, M. I., Gutiérrez-Fisac, J. L., Lostao, L., Galán, I. (2002). Desigualdades sociales en salud: situación en España en los últimos años del siglo XX. 1 a ed. Murcia: Universidad de Alicante, Sede Universitaria de Cocentaina.

Rogers, R. G, y Hackenber, R. (1987). Extending epidemiologic transition theory. En Social Biology, 34, 234-243.

Schoenbach, V., Klein, R. y Fried, R. (2000). Standarization. Recuperado de www.sph.unc.edu/courses/EPID168.

Vallin, J. y Mesle, F. (2010). De la transition epidemiologique a la Transition sanitarie: l'improbable convergence generale. En Chaire Quetelet. Bélgica: Universidad Catholique de Louvaine.

\section{Reconocimiento}

Este artículo se enmarca en el proyecto financiado por el Ministerio de Economía y Competitividad español con número de referencia CSO2012-31206, titulado: Relaciones dinámicas entre mortalidad y fecundidad en las primeras fases de la Transición Demográfica, en el cual está como investigadora Margarita Gonzalvo-Cirac, autora principal de este artículo. 
Tabla 1: Esperanza de vida al nacer y diferencia por sexo, 1981-2011

\begin{tabular}{ccccccccccc}
\hline \multirow{2}{*}{ Año } & \multicolumn{3}{c}{ Hombres } & \multicolumn{3}{c}{ Mujeres } & \multicolumn{2}{c}{ Diferencia entre sexos } \\
& España & Cataluña & Tarragona & España & Cataluña & Tarragona & España & Cataluña & Tarragona \\
\hline $\mathbf{1 9 8 1}$ & 72,39 & 74,55 & 72,96 & 78,52 & 78,74 & 78,20 & 6,13 & 4,19 & 5,24 \\
$\mathbf{1 9 9 1}$ & 73,40 & 73,80 & 73,88 & 80,44 & 80,66 & 80,30 & 7,04 & 6,86 & 6,42 \\
$\mathbf{2 0 0 1}$ & 75,89 & 76,18 & 76,15 & 82,74 & 83,01 & 82,53 & 6,85 & 6,83 & 6,38 \\
$\mathbf{2 0 0 5}$ & 77,00 & 77,28 & 77,03 & 83,60 & 83,79 & 83,15 & 6,60 & 6,51 & 6,12 \\
$\mathbf{2 0 1 1}$ & 78,70 & 78,95 & 78,45 & 84,70 & 84,95 & 84,70 & 6,00 & 6,00 & 6,25 \\
\hline
\end{tabular}

Fuente: Elaboración propia a partir de tablas de mortalidad para este período.

Tabla 2: Evolución de la esperanza de vida a la edad de 65 años por sexo

\begin{tabular}{ccccccc}
\hline \multirow{2}{*}{ Año } & \multicolumn{3}{c}{ Hombres } & \multicolumn{3}{c}{ Mujeres } \\
& España & Cataluña & Tarragona & España & Cataluña & Tarragona \\
\hline $\mathbf{1 9 8 1}$ & 15,42 & 15,60 & 16,10 & 19,07 & 19,50 & 19,29 \\
$\mathbf{1 9 9 1}$ & 16,08 & 16,30 & 16,58 & 19,97 & 20,60 & 20,00 \\
$\mathbf{2 0 0 1}$ & 16,61 & 16,80 & 17,10 & 20,59 & 21,10 & 20,61 \\
$\mathbf{2 0 0 5}$ & 17,30 & 17,17 & 17,33 & 21,40 & 21,00 & 20,81 \\
$\mathbf{2 0 1 1}$ & 18,09 & 18,31 & 18,17 & 22,01 & 22,18 & 21,91 \\
\hline
\end{tabular}

Fuente: Elaboración propia a partir de las tablas de mortalidad para este período.

Tabla 3: Porcentaje de defunciones por grandes causas de muerte. Tarragona, 1991, 2001, 2011

\begin{tabular}{lrrr}
\hline Causa de muerte & $\mathbf{1 9 9 1}$ & $\mathbf{2 0 0 1}$ & $\mathbf{2 0 1 1}$ \\
\hline Enfermedades infecciosas & 2,03 & 1,78 & 1,70 \\
Cáncer-tumores & 22,14 & 24,71 & 25,28 \\
Enfermedades del aparato circulatorio & 36,64 & 32,12 & 30,56 \\
Enfermedades del aparato respiratorio & 12,16 & 10,94 & 10,94 \\
Enfermedades del aparato digestivo & 5,03 & 5,09 & 4,86 \\
Enfermedad puerperal & 0,00 & 0,01 & 0,00 \\
Debilidad congénita & 1,00 & 0,29 & 0,30 \\
Perinatales & 0,00 & 0,31 & 0,33 \\
Enfermedades mal definidas & 5,31 & 3,73 & 4,34 \\
Muertes violentas & 9,37 & 5,32 & 4,89 \\
Conjunto "otras enfermedades" & 6,32 & 15,70 & 16,80 \\
\hline TOTAL & $\mathbf{1 0 0 , 0 0}$ & $\mathbf{1 0 0 , 0 0}$ & $\mathbf{1 0 0 , 0 0}$ \\
\hline
\end{tabular}

Fuente: Elaboración propia a partir de los datos del Instituto Nacional de Estadística (INE). 
Tabla 4: Índice de Mortalidad Estandarizado, Tarragona con respecto al patrón español

\begin{tabular}{lrrrrrr}
\hline Causa de muerte & \multicolumn{2}{c}{$\mathbf{1 9 9 1}$} & \multicolumn{2}{c}{$\mathbf{2 0 0 1}$} & \multicolumn{2}{c}{$\mathbf{2 0 1 1}$} \\
& Hombres & Mujeres & Hombres & Mujeres & Hombres & Mujeres \\
\hline Infecciosas-parasitarias & 0,63 & 0,81 & 0,90 & 0,80 & 0,90 & 0,80 \\
Cáncer-tumores & 0,62 & 0,65 & 0,90 & 0,70 & 0,90 & 0,80 \\
Circulatorias & 0,67 & 0,67 & 0,70 & 0,90 & 0,90 & 0,70 \\
Respiratorias & 0,64 & 0,56 & 0,90 & 0,80 & 0,90 & 1,01 \\
Digestivas & 0,60 & 0,66 & 0,70 & 0,80 & 0,80 & 0,80 \\
Puerperal & $\mathrm{NA}$ & 0,68 & $\mathrm{NA}$ & 0,70 & $\mathrm{NA}$ & 0,80 \\
Anomalías congénitas & 0,82 & 0,73 & 0,70 & 0,80 & 0,90 & 0,80 \\
Perinatales & 0,54 & 0,53 & 0,70 & 0,80 & 0,90 & 0,80 \\
Causas externas & 0,75 & 1,07 & 1,14 & 1,15 & 1,10 & 1,05 \\
Otras enfermedades & 1,31 & 1,38 & 1,24 & 1,23 & 1,20 & 1,22 \\
\hline TOTAL & $\mathbf{0 , 7 1}$ & $\mathbf{0 , 7 5}$ & $\mathbf{1 , 0 1}$ & $\mathbf{0 , 9 0}$ & $\mathbf{1 , 0 1}$ & $\mathbf{1 , 0 0}$ \\
\hline Funn
\end{tabular}

Fuente: Elaboración propia. 